\title{
Exacerbation of Cardiovascular Complications in case of Covid-19 Patients and their Treatments
}

\author{
Mst. Mahfuza Rahman ${ }^{1}$, Uthpall Kumar Roy $^{1}$, Md. Kouser ${ }^{1}$, Shahriar Mohammad Shohan ${ }^{1}$, Sangita \\ Chakraborty $^{1}$, and Mir Imam Ibne Wahed ${ }^{2}$
}

${ }^{1}$ Department of Pharmacy, Comilla University, Koatbari, Cumilla-3506, Bangladesh; ${ }^{2}$ Department of Pharmacy, University of Rajshahi, Rajshahi-6205, Bangladesh.

*Correspondence: mahfuzarahman49@gmail.com (Mst. Mahfuza Rahman, Lecturer, Department of Pharmacy, Comilla University, Koatbari, Cumilla-3506, Bangladesh).

\section{ABSTRACT}

The 2019 Corona virus Outbreak (COVID-19) is a scientific, medical and social challenge. The complexity of Severe Acute Respiratory Syndrome Corona virus 2 (SARSCoV2) focuses on the clinical course of unpredictable illnesses that can develop rapidly and cause serious complications leading to death. Systemic inflammation and lung problems can ensue, causing considerable morbidity and mortality. Acute coronary syndrome (ACS), arrhythmias, myocarditis, acute myocardial damage, heart failure, and other problems affect the cardiovascular system. Existing data about cardiovascular complications had been collected from the case study performed in China, Wuhan and New York COVID-19 patients. In the case report of China, $16.7 \%$ out of 138 patients showed arrhythmias where heart failure was identified as a problem in $23 \%$ of patients in a retrospective study from Wuhan and 52\% of non-survivors in China. On the other hand case studies on 18 COVID-19 patients with ST-elevation myocardial infarction (STEMI) on electrocardiogram (ECG) from New York were investigated; 6 patients(33\%) reported chest pain, 14 patients (78\%) reported ST-segment elevation, 6 patients $(35 \%)$ reported regional wall motion abnormality on TTE, and 8 patients (44\%) reported a clinical diagnosis of myocardial infarction and a total of 9 patients individuals $(50 \%)$ had coronary angiography, with 6 patients $(67 \%)$ of them showing obstructive disease. The cardiovascular consequences of COVID-19 infection are examined in this brief paper. The virus attaches to ACE2 (Angiotensin converting enzyme 2), allowing it to enter. COVID-19 therapy is currently being studied in conjunction with cardiovascular drugs. Therefore, emergency physicians should keep these cardiovascular complexities in mind while assessing and treating patients with COVID-19.

Keywords: COVID-19, Acute coronary syndrome, Heart failure, Myocarditis, and acute myocardial injury.

\section{INTRODUCTION:}

Corona virus disease 2019 (COVID-19) is a new, rapidly spreading infectious disease of the human respiratory system. It is caused by a recently discovered enveloped RNA $\beta$ corona virus called severe acute respiratory syndrome coronavirus 2 (SARSCoV2) (Lu et al., 2020). SARSCoV2 is the seventh known coronavirus that infects humans. The coronavirus has contributed to previous epidemics such as SARS-CoV and MERS-CoV. In December 2019after a case study of indicators related to the fishery goods and wet animal whole-sale market in Wuhan, Hubei Province, China, it extended to more than 200 countries (Callaway et al., 2020). As of 09:21 UTC on 28 July 2021 , there are $195,368,552$ cases that have been confirmed and 4,178,287 deaths in almost 200 countries/territories and 26 cruise/naval ships (Mallapaty, 2020).

Patients with existing companion illnesses, especially CVD, are at high risk of serious illness. COVID-19 can cause terrifying pulmonary complications such as 
acute respiratory distress syndrome, as well as acute myocardial damage and dysfunction, which can promote or contribute to shock and manifold organ failure. In this study, the effects of COVID 19 on the $\mathrm{CV}$ system are divided into primary or secondary cardiac invasion. Arrhythmias, acute coronary syndrome (ACS), and myocarditis are the most common cardiac symptoms of COVID19 illness. Secondary cardiac invasion is commonly caused by systemic inflammatory syndrome and may be manifested in acute myocardial damage/ elevated biomarkers and / or heart failure (CHF). Secondary cardiac intervention often includes other evidence of visceral damage. Finally, additional vascular complications of COVID19 disease are identified and described (Dan et al., 2020).

\section{MATERIALS AND METHODS:}

Strategy Searching - According to the pre-determined protocol a systematic literature search was conducted in PubMed, Google Scholar, SCOPUS databases from $1^{\text {st }}$ July, 2021 to $1^{\text {st }}$ August, 2021. Symptoms of COVID-19, heart disease, COVID-19 fatalities, cardiovascular abnormalities, and myocardial injury, causes of myocardial infarction, arrhythmia, and acute coronary syndrome were search terms for the literature searching function (Long et al., 2020). We didn't look for preprints on websites since several experts were concerned about the quality of some articles before they went through a rigorous peer-review procedure (Impact of Social Sciences, 2020).

Study Selection - Six reviewers did the literature search and exported the papers that were found to be eligible. The literature was screened based on the desired outcome. Approximately 55 articles were primarily retrieved a total of 36 articles were selected that fulfilled the stated review criteria for inclusion. All conflicts were resolved by all the reviewers.

Data Information - Our review covered trials that involved patients who were at least 18 years old. The major outcome of interest was cardiovascular complications with severe COVID-19, which was defined as all-cause death, ICU hospitalization, ARDS, or mechanical ventilation requirement. Pre-existing CVD and its conventional risk variables identified in major CVD clinical guidelines like age, sex, smoking, hypertension, dyslipidemia, and diabetes were all potential risk factors of interest (Karmali and Lloyd Jones, 2017).
Data Extraction - Full-text papers were checked and the following data was extracted like year of publication, study date, sample size of COVID-19 patients, number of participants with each co morbidity who died and did not die, cardiovascular complications in case of Covid-19 patients, the mean or median age, as well as their standard deviations and the pro-portion of men in each study.

Data Synthesis and Analysis - The number of studies, study settings, proportion of sex, means or median age, and variables accounted for in each research were all described using a narrative method. The risk of death in COVID-19 individuals with preexisting cardiovascular illnesses was our primary outcome. However, reviewers visually evaluated for the likelihood of publication bias, and heterogeneity of study estimates was assessed to maintain the possibility of significant heterogeneity (Sentongo et al., 2020).

\section{RESULTS:}

COVID-19 and cardiovascular disease - COVID19 's influence on the cardiovascular system will be split into main and secondary cardiac involvement in this review; there is, of course, a lot of overlap between the two. Arrhythmias, ACS, and myocarditis are the primary cardiac symptoms of COVID-19 illness. A systemic inflammatory condition can cause seconddary cardiac involvement, which can appear as acute myocardial damage, biomarker increase, and/or heart failure (CHF). Other signs of end-organ dam-age are frequently seen with secondary cardiac involvement.

\section{Primary Cardiac Involvement}

Arrhythmias - COVID-19 has been shown to have both tacky- and brady-arrhythmias. Research that looked at the clinical characteristics and prognosis of 138 Chinese patients with COVID-19 found that $16.7 \%$ of them had arrhythmia (Wang et al., 2020). Additionally, patients receiving hydroxy-chloroquine treatment should consider the warnings listed below(1) dosage decrease for individuals with severe renal insufficiency (50 percent for creatinine clearance 10 $\mathrm{mL} / \mathrm{min}$ ), (2) vigorous electrolyte correction prior to usage, and (3) avoidance or cautious monitoring for patients with hereditary long QT or those taking several QT prolonging medicines (Ayad et al., 2010; Chen et al., 2020). The high rate of hypokalemia in COVID-19 patients may, at least in part, increase the risk of arrhythmia (Chen et al., 2020). SARS-CoV-2 
infects cells via attaching to ACE2 receptors, which increases angiotensin II availability and therefore increases urine potassium excretion (Chen et al., 2020).

Acute Coronary Syndrome (ACS) - ACS is more common in the presence of a virus, most likely owing to plaque instability caused by inflammation. Although the risk of COVID-19 infection is unclear, other viruses have been linked to a three- to tenfold increase in risk (BOYLE, 1997; Naghavi et al., 2003; Gattone et al., 2001). In the midst of the global crisis, several healthcare facilities in Europe, North America, and Asia have reexamined their processes for treating ACS, taking into account the potential dangers of healthcare worker exposure and the usage of personal protective equipment. In a case series from New York, 18 COVID-19 patients with STEMI on ECG were investigated; 6 (33\%) of these patients reported chest pain, 14 (78\%) had localized ST-segment elevation, $6(35 \%)$ had a regional wall motion abnormality on TTE, and $8(44 \%)$ had a clinical diagnosis of myocardial infarction (Bangalore et al., 2020). A total of $9(50 \%)$ individuals had coronary angiography, with $6(67 \%)$ of them showing obstructtive disease (Bangalore et al., 2020). Infection control and management are two additional factors that may influence the therapeutic approach and therapy of ACS in patients with COVID-19 (Nahid, 2021).

Myocarditis - COVID-19 acute myocarditis has an unclear actual prevalence. Although few occurrences of clinically diagnosed myocarditis have been documented in COVID-19 individuals, the number of autopsy and endomyocardial biopsy (EMB) confirmed cases is low. One case from Italy revealed widespread myocardial edema and EMB on cardiovascular magnetic resonance imaging (CMR), as well as diffuse T-lymphocytic inflammatory infiltrates (Sala et al., 2020). SARS-CoV-2 was found in cardiac macrophages but not cardiomyocytes in another instance, according to an EMB (Mihic et al., 2015). These findings support the hypothesis that myocarditis is a source of cardiac damage in COVID-19 patients, although the exact mechanism of myocarditis remains unclear. EMB and CMR have little value in the treatment of these individuals. Multiple antiviral (e.g., remdesivir, ribavirin, lopinavir, hydroxylchloroquine) and anti-inflammatory (e.g., interferon, corticosteroids, tocilizumab, sarilumab, siltuximab, anakinra, intravenous immunoglobulin, statin) treatments are being investigated to help us manage UniversePG I www.universepg.com
COVID-19 myocarditis patients (Zhou et al., 2020). Hydroxychloroquine in a well-designed retrospective study, 9 more recently has shown poor efficacy for the treatment of COVID-19 disease (Porfidia et al., 2021).

\section{Secondary Cardiac Involvement}

Acute Myocardial Injury - The most prevalent CV consequence in COVID-19 is acute myocardial infarction. Acute myocardial damage has been defined differently in different studies, such as an increase in cardiac enzymes or electrocardiographic abnormallities. In COVID-19 disease, the etiology of acute myocardial damage is likely complex, with many overlapping path-ways (Mihic et al., 2015). Acute myocardial dam-age has been observed in around a quarter of hospitalized patients, with greater cardiac troponin levels indicating a more serious condition (Zhou et al., 2020; Guo et al., 2020, Lippi, Lavie and Sanchis-Gomar, 2020). One retrospective research found that COVID-19 non-survivors had fast troponin increase late in their course, indicating that a local troponin test, in addition to CRP, D-dimer, and NT-proBNP, may be utilized to follow clinical progress (Zhou et al., 2020). Acute cardiac dam-age has regularly been demonstrated to be a powerful unfavorable prognostic marker in patients with COVID19 (Wang et al., 2020; Huang et al., 2020), regardless of the actual frequency. However acute plaque rupture and viral invasion are another two mechanisms that cause direct myocardial damage. According to recent histopathology evidence COVID-19 endotheliitis is caused by direct endothelial viral invasion with diffuse endothelial inflammation (Varga et al., 2020). This Endotheliitis is known to cause tissue inflammation and organ ischemia by impairing microcirculatory activity (Bonetti et al., 2003). Furthermore, in the setting of COVID-19 infect-ion, individuals without prior cardiac illness have presented with mainly cardiac symptoms, reflecting a potential clinical picture of myocarditis (Hu et al., 2020; Karmpaliotis et al., 2007). The inflammatory reaction and cytokine storm seen in severe COVID-19 illness may exacerbate myocardial stress and damage. This can affect heart function indirectly via peripheral effects on systemic vascular resistance (Pelliccia et al., 2017).

Heart Failure - Heart failure can happen due to myocardial injury, and chronic stable heart failure is liable to worsen during COVID-19 infection. It is not un- 
usual for people with COVID-19 illness to have new heart failure (HF). Heart failure was identified as a problem in $23 \%$ of patients in a retrospective study from Wuhan, China (52\% of non-survivors) (Zhou et al., 2020). Heart failure in COVID-19 patients can range from mild heart failure with a normal ejection fraction early on in the disease to severe heart failure with a reduced ejection fraction later on in the illness to severe end-stage heart failure and cardiogenic shock with a high mortality risk. Patients with underlying heart failure have an increased risk of mortality and morbidity (Porfidia et al., 2021; Mehra and Ruschitzka, 2020). The actual cause of COVID-19's ventricular failure is unknown. In individuals with AR-DS, higher levels of serum brain natriuretic peptide (BNP) have been related to cardiogenic pulmonary edema (Karmpaliotis et al., 2007). Surprisingly, people with COVID-19 might have elevated BNP levels even if they don't have any ventricular dysfunction (Zhou et al., 2020). Nonetheless, increased cardiac biomarkers, especially troponin, should prompt clinical suspicion of heart failure. However, because there are numerous causes of heart damage, interpreting cardiac biomarkers can be difficult. If heart failure is suspected, a restricted TTE or POCUS can measure biventricular function.

\section{Treatment}

ACEIs/ARBs/Angiotensin Receptor-Neprilysin Inhibitor (ARNIs) - Patients with heart failure and a decreased ejection fraction, independent of COVID19 infection, should be treated with an angiotensin converting enzyme inhibitor or angiotensin receptor blocker while being closely monitored (Porfidia et al., 2021). Because sacubitril concentration rises in parallel with ritonavir administration, it's critical to keep track of blood pressure, serum creatinine, and potassium levels in patients on sacubitril/valsartan.

Beta-Blockers - Patients with ACS, with the exception of those with hemodynamic instability and pulmonary edema, and those with heart failure with a decreased ejection fraction, should be treated with a beta-blocker. Carvedilol is metabolized via glucuronicdation (UGT1A1) and CYP450, metoprolol is processed mostly by CYP2D6, and bisoprolol is partly metabolized by CYP3A4 and CYP2D6 (Giguère et al., 2019). As a result, ritonavir can alter the metabolism of all of these drugs, which is why patients on lopinavir/ritonavir should have their heart rate and blood pressure closely monitored.
Diuretics - Loop diuretics and anti-COVID medicines have no known interactions, thus they can be started or sustained safely in individuals with COVID-19. Because these groups of patients have a significant risk of acute renal damage, diuretic medication should be continuously evaluated in terms of vital signs, serum electrolytes, and creatinine. Mineral-ocorticoid antagonists can be continued in individuals with COVID 19 provided they are given under strict supervision.

Digoxin - Because of the risk of instability during an acute infection, all patients on digoxin who are hospitalized with COVID-19 should be closely watched. This may demand serum concentration measurements in COVID-19 patients who continue to take digoxin. Furthermore, ritonavir can enhance the AUC and half-life of digoxin by $29 \%$ and $43 \%$, respectively. Hydroxychloroquine can also raise digoxin levels through an unidentified mechanism (Dixon et al., 2020).

Hydralazine/Nitrates - Protease inhibitors can lower the amount of nitric oxide produced by isosorbide dinitrate, reducing its effectiveness. Protease inhibitors and hydralazine have a strong interaction. Dose modifications should be made based on the patient's reaction (Giguère et al., 2019).

Ivabradine - Despite a maximum acceptable dose of beta-blockers, patients with ejection fractions of 35 per-cent or below, sinus rhythms, and minimal resting heart rates of 70 beats per minute are treated with ivabradine, and I(f) channel blocker. Ivabradine appears to be safe to continue in COVID-19 individuals who are hemodynamically stable. Ivabradine's AUC can be increased by six-fold when combined with strong CYP450 inhibitors; therefore, it should be avoided in patients taking lopinavir/ritonavir for COVID-19 (Lippi et al., 2020).

\section{DISCUSSION:}

Principal findings - In this systematic review, we focus on the link between severe COVID-19 and CVD and its associated risk factors. The findings of this systematic review show that patients with preexisting cardio-vascular illness, hypertension, congestive heart failure, chronic renal disease, and patients who are hospitalized with COVID-19 have a higher risk of mortality from COVID-19. In studies that controlled for age and a few other co-morbidities, one very large research found independent correlations of hypertension with severe COVID-19 (Liang 
et al., 2020). The independence of these relationships from significant confounders, notably age, is a key concern. However, the observed correlations of hypertension and diabetes with the severity of COVID19 infection should not be interpreted as evidence of renin-angiotensin system inhibitor side effects. Furthermore, in the sole research that looked at the prevalence of renin-angiotensin system inhibitor usage (Cheng et al., 2020), 30\% of patients had hypertension, but only $5 \%$ of them were using reninangiotensin system inhibitors (Cheng et al., 2020). As a result, several expert groups' advice continuing to use this class of medications until further data becomes available (South et al., 2020).

\section{Consequences for clinical practice and research -}

Our findings support the concept that people who appear to be at higher risk should be given vaccine first to develop immunization against SARS-CoV2.Individuals with pre-existing cardiovascular illness, hypertension, diabetes, congestive heart failure, chronic renal disease, and cancer are at a higher risk of mortality from the virus and should be given first priority when a vaccine is distributed, especially if supplies are limited. Individuals with pre-existing cardiovascular illness, hypertension, diabetes, congestive heart failure, chronic renal disease, and cancer are at a higher risk of mortality from the virus and should be given first priority when a vaccine is distributed, especially if supplies are limited. SARSCoV-2 may become seasonal, necessitating yearly immunization, according to mounting data (Moriyama et al., 2020; Islam et al, 2021).

Strength - Our systematic review offers a number of advantages: a comprehensive assessment of CVD and its risk factors, clinically appropriate criteria of severe COVID-19 that reduce subjective reporting, and careful consideration of probable patient overlap using restricted and inclusive meta-analyses. Meta-regression was used to investigate possible con-founding, and the period between the literature search and paper submission was only 3 weeks.

\section{Limitations of the study}

The first constraint is that the bulk of researches were undertaken in China because the pandemic started there. But we must have to recognize the geographical differences in some risk variables, and additional research in many locations (Wang et al., 2019). Secondly, we did not include non-English publiccations and so overlooked several important studies.
Thirdly the majority of research used odds ratios, which are known to overestimate risk ratios when exposure prevalence is high. Finally, it's impossible to rule out the likelihood that some patients were included in several researches, particularly the China CDC report and other Chinese investigations (Liu et al., 2020).

\section{CONCLUSION:}

COVID-19's widespread distribution has wreaked havoc on people's health all over the world. COVID19 is now recognized to cause cardio-vascular problems, and persons with underlying cardiovascular disease are more vulnerable to infection. As this new epidemic unfolds, a number of cardiac symptoms are becoming apparent. It's important to note that in the post-pandemic age, cardiovascular problems will still be the primary cause of mortality. At this time, there is unquestionably a dearth of information on the cardiovascular implications, thus more study is needed to identify the safest drug regimen for COVID-19 patients. In order to avoid or effectively manage COVID-19 infection-related consequences, patients with hypertension should be monitored on a regular basis. Clinicians should be aware of the known cardiac involvement and address it at the onset to minimize harm until a vaccine or potentially curative treatment is available. We will need a longerterm follow-up of severe COVID-19 patient survivors due to the increasing recognition of CVD damage in this disease. In addition, we need to be aware of the negative effects and drug interactions that now exist.

\section{ACKNOWLEDGEMENT:}

First and foremost, the author is grateful to Almighty Allah. The author is also thankful to anonymous reviewers and editors for their supportive comments and suggestions.

\section{CONFLICTS OF INTEREST:}

The authors declare that they have no competing interests.

\section{REFERENCES:}

1) Ayad et al. (2010). July. Causes and management of drug-induced long QT syndrome. In Baylor University Medical Center Proceedings, Taylor \& Francis, 23(3), pp. 250-255. https://doi.org/10.1080/08998280.2010.11928628

2) Bangalore et al. (2020). ST-segment elevation in patients with Covid-19a case series. New 
England J. of Medicine, 382(25), pp. 24782480. https://doi.org/10.1056/NEJMc2009020

3) Bonetti et al. (2003). Endothelial dysfunction: a marker of atherosclerotic risk. Arteriosclerosis, thrombosis, and vascular biology, 23(2), pp. 168-175.

https://doi.org/10.1161/01.ATV.0000051384.431 $\underline{04 . \mathrm{FC}}$

4) Boyle, J. J. (1997). Association of coronary plaque rupture and atherosclerotic inflamemation. The Journal of Pathology: A Journal of the Pathological Society of Great Britain and Ireland, 181(1), pp.93-99.

5) Callaway et al. (2020). The coronavirus pandemic in five powerful charts. https://doi.org/10.1038/d41586-020-00758-2

6) Cheng et al. (2020). Kidney impairment is associated with in-hospital death of COVID-19 patients. MedRxiv. https://doi.org/10.1101/2020.02.18.20023242

7) Dan et al. (2020). The Case Fatality Rate in COVID-19 Patients with Cardiovascular Disease: Global Health Challenge and Paradigm in the Current Pandemic. Current Pharmacology Reports.

https://link.springer.com/article/10.1007/s40495020-00239-0

8) Dixon et al. (2020). Cardiovascular considerations in treating patients with coronavirus disease 2019 (COVID-19). Journal of cardiovascular pharmacology.

https://link.springer.com/article/10.1097/FJC.000 $\underline{0000000000836}$

9) Gattone et al. (2001). Chlamydia pneumoniae and cytomegalovirus seropositivity, inflamematory markers, and the risk of myocardial infarction at a young age. American heart journal, 142(4), pp.633-640.

10) Giguère et al. (2019). Getting to the heart of the matter: a review of drug interactions between HIV antiretrovirals and cardiology medications. Canadian Journal of Cardiology, 35(3), pp.326-340.

11) Guo et al. (2020). Cardiovascular implications of fatal outcomes of patients with coronavirus disease 2019 (COVID-19). JAMA cardiology, 5(7), pp.811-818. https://doi.org/10.1001/jamacardio.2020.1017

12) Heimstädt, M. (2020). Between fast science and fake news: Preprint servers are political. Impact of Social Sciences Blog.
13) Huang et al. (2020). Clinical features of patients infected with 2019 novel corona-virus in Wuhan, China. The lancet, 395(10223), pp.497506.

https://doi.org/10.1016/S0140-6736(20)30183-5

14) $\mathrm{Hu}$ et al. (2021). Coronavirus fulminant myocarditis treated with glucocorticoid and human immunoglobulin. European heart journal, 42 (2), pp.206-206. https://doi.org/110.1093/eurheartj/ehaa190

15) Islam MS, Dayem SB, and Amin M. (2021). Impact of host genetic players on Covid-19 disease severity: a review of current knowledge and future prospect, Bangladesh. Eur. J. Med. Health Sci., 3(4), 79-87. https://doi.org/10.34104/ejmhs.021.079087

16) Karmali, K.N. and Lloyd Jones, D.M. (2017). Implementing cardiovascular risk prediction in clinical practice: the future is now.

https://www.ahajournals.org/doi/full/10.1161/JA HA.117.006019

17) Karmpaliotis et al. (2007). Diagnostic and prognostic utility of brain natriuretic Peptide in subjects admitted to the ICU with hypoxic respiratory failure due to noncardiogenic and cardiogenic pulmonary edma. Chest, 131(4), pp.964-971.

https://doi.org/10.1378/chest.06-1247

18) Li et al. (2020). Hypokalemia and clinical implications in patients with coronavirus disease 2019 (COVID-19). MedRxiv.

19) Liang et al. (2020). Cancer patients in SARSCoV-2 infection: a nationwide analysis in China. The lancet oncology, 21(3), pp.335-337. https://www.ahajournals.org/doi/full/10.1001/jam aoncol.2020.6778

20) Lippi et al. (2020). Cardiac troponin I in patients with coronavirus disease 2019 (COVID19): evidence from a meta-analysis. Progress in cardiovascular diseases, 63(3), p.390. https://doi.org/10.1016/j.pcad.2020.03.001

21) Liu et al. (2020). Influenza activity during the outbreak of coronavirus disease 2019 in Chinese mainland. Biosafety and Health, 2(4), pp.206-209.

22) Long et al. (2020). Cardiovascular complications in COVID-19. The American journal of emergency medicine, 38(7), pp.1504-1507.

23) Lu et al. (2020). Genomic characterization and epidemiology of 2019 novel coronavirus: im- 
plications for virus origins and receptor binding. The lancet, 395(10224), pp.565-574.

24) Mallapaty, S. (2020). What the cruiseship outbreaks reveal about COVID-19. Nature, 580 (7801), pp.18-19. https://doi.org/10.1038/d41586-020-00885-w

25) Mehra, M.R. and Ruschitzka, F. (2020). COVID-19 illness and heart failure: a missing link? https://doi.org/10.5167/uzh-201064

26) Mihic et al. (2015). A conductive polymer hydrogel supports cell electrical signaling and improves cardiac function after implantation into myocardial infarct. Circulation, 132(8), pp.772-784.

27) Moriyama et al. (2020). Seasonality of respiretory viral infections. Annual review of virology, 7, pp.83-101.

28) Naghavi et al. (2003). Influenza infection exerts prominent inflammatory and thrombotic effects on the atherosclerotic plaques of apolipoprotein E-deficient mice. Circulation, 107(5), pp.762-768. https://doi.org/10.1161/01.CIR.0000048190.6807

29) Nahid AHM. (2021). Resistance, unrest, protest and demonstration provoked by COVID-19: a case study of Bangladesh, Asian J. Soc. Sci. Leg. Stud., 3(2), 23-34. https://doi.org/10.34104/ajssls.021.023034

30) Pelliccia et al. (2017). Pathophysiology of Takotsubo syndrome. Circulation, 135(24), pp. 2426-2441.

https://doi.org/10.1161/CIRCULATIONAHA.116. $\underline{027121}$

31) Porfidia et al. (2021). CT Pulmonary Angiography for the Diagnosis of Pulmonary Embolism in Patients with COVID-19: When, why, and for whom? Radiology, 299(3), pp. E287E287. https://doi.org/10.1148/radiol.2021210400
32) Sala et al. (2020). Acute myocarditis presenting as a reverse Tako-Tsubo syndrome in a patient with SARS-CoV-2 respiratory infection. European heart journal, 41(19), pp.1861-1862. https://doi.org/10.1093/eurheartj/ehaa286

33) South et al. (2020). Controversies of reninangiotensin system inhibition during the $\mathrm{CO}$ VID-19 pandemic. Nature Reviews Nephrology, 16(6), pp.305-307. https://doi.org/10.1038/s41581-020-0279-4

34) Ssentongo et al. (2020). Association of cardiovascular disease and 10 other pre-existing comorbidities with COVID-19 mortality: A systematic review and meta-analysis. PloS one, 15(8), pp.e0238215. https://doi.org/10.1371/journal.pone.0238215

35) Varga et al. (2020). Endothelial cell infection and endotheliosis in COVID-19. The Lancet, 395(10234), pp.1417-1418. https://doi.org/10.1016/S0140-6736(20)30937-5

36) Wang et al. (2019). Trends in smoking prevalence and implication for chronic dis-eases in China: serial national cross-section-al surveys from 2003 to 2013. The Lancet Respiratory Medicine, 7(1), pp.35-45.

37) Wang et al. (2020). Clinical characteristics of 138 hospitalized patients with 2019 novel coronavirus infected pneumonia in Wuhan, China. Jama, 323(11), pp.1061-1069. https://doi.org/10.1001/jama.2020.1585

38) Zhou et al. (2020). Clinical course and risk factors for mortality of adult inpatients with COVID-19 in Wuhan, China: a retrospective cohort study. The lancet, 395(10229), pp.10541062 . https://doi.org/10.1016/S0140-6736(20)30566-3 Med. Health Sci., 3(5), 116-122. https://doi.org/10.34104/ejmhs.021.01160122 @) () 